\title{
REPOBLAMIENTO GRADUAL Y CONTROLADA DEL CAMARÓN (Cryphiops caementarius) EN LOS RÍOS SAMA Y LOCUMBA DE TACNA
}

Cornelio Valencia Yauli ${ }^{1}$

\author{
$R E S U M E N$
}

\begin{abstract}
El Proyecto de Investigación fue realizado en los ríos Sama y Locumba de Tacna, entre enero y diciembre de 1997, a fin de efectuar la repoblación con post larvas de Camarón de río (Cryphiops caementarius) y atenuar la extinción de una especie nativa de importancia económica.

Durante el trabajo de campo se realizó la captura masiva de post larvas (talla entre 1,5 a 4,0 cm), acopio, movilización, resiembra y evaluación preliminar. También se ejecutaron controles físico químicos del agua, densidad y tiempo de transporte, mortalidad existente, controles de talla y peso de la población transportada y evaluación final bioecológica del recurso introducido en el hábitat.

Durante el proyecto se logró transportar una cantidad total de 17300 semillas de Camarón de río (Cryphiops caementarius); 14000 fueron introducidas en el río Sama y 3300 especímenes en el ríc Locumba.
\end{abstract}

\section{A B S TR A C T}

The Project of Investigation was carried out in the rivers Sama and Locumba of Tacna, between January and December of 1997, in order to carryout the repopulation with river Shrimp at a post-larval stage (Cryphiops caementarius) and to attenuate the extinction of a native species of economic importance.

During the work on site the massive capture of post larvas was carried out (measuring from 1,5 to $4,0 \mathrm{~cm}$ ), gathering, mobilization, replacement and preliminary evaluation. Physical and chemical tests of the water, density and time of transport were also executed as well as existent mortality, control of size and weight of the transported population, and a final bioecologica evaluation of the resource introduced in the habitat.

During the project it was possible to transport a total quantity of 17300 seeds of river Shrimp (Cryphiops caementarius); 14000 were introduced to the river Sama and 300 specimins in the river Locumba.

\section{INTRODUCCIÓN}

El desarrollo técnico Industrial de los países en los últimos años ha generado beneficios y comodidades a la población humana, este avance a su vez, genera el incremento del grado de contaminación en el medio ambiente, principalmente en los ríos y aguas marinas cercanos a las urbes, por ser depositarios de diversos desechos o residuos sólidos procedentes de las industrias y de la misma población. 
La contaminación de los cuerpos lóticos es la más factible y la que con mayor incidencia se presenta, su alteración genera problemas de desaparición y extinción bioecológica de diferentes organismos acuáticos (flora y fauna típica de un ambiente). El decremento del stock poblacional de camarones en el medio acuático está influenciado también por factores como contaminantes hidro metálicos, desechos mineros metalúrgicos, residuos de productos agro industriales, contaminación de aguas servidas, etc.

Procesos hidromecánicos de tipo similar se presentan en el río Locumba (Tacna), recurso hídrico que es utilizado como colector final de los relaves mineros procedentes de los complejos de Toquepala y Cuajone(S.P.C.C.). Esta polución hidrocontaminante de los residuos minero - metalúrgicos ha ocasionado la extinción total del Camarón de río (Cryphiops caementarius) en toda la cuenca del río. Asímismo poblaciones camaroneras del río Sama han disminuído notablemente por la excesiva extracción del crustáceo (sobre pesca) y la presencia de factores adversos en la climatología limnética.

Una de las alternativas para volver a tener la presencia de camarones en estos ríos lo constituye la repoblación biológica de las aguas con semillas de camarón nativo, especímen propio de estos ambientes. El objetivo del trabajo es desarrollar e implementar una metodología técnica práctica, adecuada para efectuár la recolección, transporte y siembra masiva del Camarón de río (Cryphiops caementarius) en la cuenca de los ríos Sama y Locumba.

La "semilla" se refiere a camarones en estado post larval o juveniles, especímenes vivos que sirven para formar una biopoblación acuática, reemplazante de organismos en proceso de extinción, dentro de una biota conocida.

Generalmente las operaciones de repoblación son necesarias cuando los crustáceos, por causas diferentes, están en proceso de desaparición del medio acuático, a consecuencia de un desequilibrio irreversible de este medio. El vacío ecológico debe ser llenado por un animal que tenga una "profesión ecológica" idéntica, con hábitos y exigencias compatibles a la del medio ambiente. La repoblación recae entonces sobre la especie indígena inexistente en el río. La carga poblacional introducida debe mantenerse en equilibrio con la capacidad de acogida del medio y de sus posibilidades alimenticias.
El Camarón de río (Cryphiops caementarius) es uno de los decápodos más conocidos y de mayor importancia económica de la Costa Occidental y Central de América Meridional. Su ámbito geográfico distribucional corresponde a la región costera del Pacífico Sur Oriental de América, correspondiente a los ambientes ecológicos peruano - chileno, entre los límites latitudinales de $06^{\circ} 32^{\prime}$ L.S. (río Taymi Pativilca) y $30^{\circ}$ L.S. (río Loa - Chile), cuya fauna acuática pertenece a las aguas templadas. La zona de mayor abundancia en el territorio peruano se presenta en los ríos Camaná, Ocoña y Majes (Arequipa).

El Camarón de río es un recurso hidrobiológico de origen dulce acuícola de zonas templadas que, en su medio natural, tiene preferencias por aguas lóticas, de corriente moderada, coloración semi transparente, con fondo areno pedregoso - fangoso y cuyas orillas mantienen vegetación acuática o ribereña; características que lo protegen de la luz, de posibles depredadores y le proporcionan refugios adecuados.

Para cumplir su ciclo biológico reproductivo, el Camarón de río (Cryphiops caementarius) requiere bajar a la zona de desembocadura de los ríos (meses de verano).

Las hembras de camarón son bastante prolíferas, alcanzando su madurez sexual antes del primer año de vida; hembras ovigeras con tallas entre 2,5 a 3,0 $\mathrm{cm}$ alcanzan a desovar entre 500 a 800 óvulos.

La alta densidad de carga durante el transporte, la temperatura inestable y la carencia de oxígeno en el medio artificial, utilizada para el transporte de las post larvas, induce a la autoeliminación masiva del camarón mediante la secreción de una sustancia tóxica semi - pastosa (color marrón amarillento) y de ciertas feromonas provenientes de la glándula verde, condiciones extremas surgidas como respuesta al stress ambiental del medio acuático.

Los camarones están sometidos a la abusiva y excesiva explotación de captura practicada por el hombre, proceso que influye directamente en el rompimiento del equilibrio biológico de la especie. Los camarones son extraídos cada día con mayor intensidad y este exceso de captura está ocasionando que la especie se encuentre en vias de extinción poblacional; debido a ello, ha sido necesario implantar períodos deveda anual para preservarsu conservación como recurso natural renovable. Este período de veda 
de los camarones comprende los meses de diciembre a marzo, generalmente épocas donde la especie se encuentra en proceso de reproducción.

\section{MATERIALES Y MÉTODOS}

\section{LUGAR Y FECHA DE REALIZACIÓN}

El trabajo de investigación se desarrolló de enero a diciembre de 1997. Es necesario precisar que en años anteriores se estuvo ejecutando y recolectando datos relacionados al proyecto, cuyos resultados están incluidos en la evaluación del presente documento.

\section{Alternativas de Ejecución}

\section{- Fase de Gabinete}

Realizado en el laboratorio de Acuicultura (EAPA)Facultad de Ingeniería Pesquera (FAIP).

\section{- Fase de Camipo}

Desarrollado en las cuencas de los ríos:

Locumba: Zona cercana a la desembocadura (Bahía de Ite) hasta los 1800 m.s.n.m.

Sama: Area de desembocadura hasta los 1400 m.s.n.m. (Coruca).

\section{ACCIONES Y TAREAS GENERALES REALIZADAS}

- Acopio y revisión bibliográfica especializada.

- Viajes de reconocimiento a la zona del proyecto.

- Análisis físicos, químicos y biológicos del agua de 02 cursos lóticos y de las áreas de captura.

- Búsqueda de lugares aparentes para extracción, recolección de semilla y siembra de post larvas.

- Métodos de extracción, captura, acondicionamiento, transporte de especímenes y mortalidad acumulada.

- Evaluación bioecológica preliminar de la siembra.

\section{METODOLOGÍA DESARROLLADA}

\section{a. De Gabinete}

\section{- Ubicación del Área de Estudio}

Comprende la ubicación geográfica del área de estudio (utilizando planos topográficos), la influencia de las zonas aledañas y la delimitación del terreno. La extensión del área de estudio fue calculada mediante el método de las coordenadas.

\section{- Estimación de la Población Requerida}

La cantidad de semilla requerida fue estimada en base a una densidad poblacional de 0,5 camarones/ $\mathrm{m}^{2}$ de rio, stock poblacional sugerida por especialistas en repoblación de camarones.

Para calcular el stock de semillas se tuvo presente las siguientes condiciones básicas:

- Las características bioecológicas de la especie.

- Cálculo de la extensión del área a repoblar y carac-terísticas hidrológicas.

- Disponibilidad y cantidad de semilla.

- Otros considerandos (costo de transporte, personal y equipos disponibles, lugar de siembra, etc.

\section{b. De Campo}

\section{- Número de viajes efectulados}

Durante los 08 meses se realizaron 05 viajes

- 01 viaje de reconocimiento de campo.

- 04 viajes de captura y transporte de semillas.

\section{- Reconocimiento del Área de Trabajo}

El trabajo de campo se inicia con el reconocimiento total del área de estudio (valle de Locumba y Sama) y las zonas ubicadas alrededor de ellos que tienen influencia con el desarrollo del trabajo.

\section{- Elección de Áreas de Captura y Áreas de Siembra}

Las zonas de captura contaban con abundante post larva de camarón y tenían acceso por carretera.

Las áreas de șiembra poseen ciertas características ecológicas: fácil acceso vehícular, pendiente ligera del terreno, abundante vegetación hidrofítica y área alejada de los agrupamientos humanos, etc. 


\section{- Captura de Semilla}

La captura se realizó empleando 2 tipos diferentes de "cal-cal" (una revestida de tela tul transparente y otra de malla anchovetera); este es un aparejo artesanal de extracción, de forma cónica y con un marco estructural de metal (tamaño $40 \times 20 \mathrm{~cm}$ ). Para el acopio de las semillas se emplean depósitos de plástico (baldes de 15 I de capacidad); en estos recipientes son trasladados al vehículo.

\section{- Transporte-Densidad}

El transporte de la semilla de camarón fue realizada utilizando cajas isotérmicas (embalajes de $35 \times 40 \times 45$ $\mathrm{cm})$. En este sistema de transporte se llena con $2 / 3$ de agua $(30 \mathrm{I})$ y una densidad prefijada de semilla (según la talla del camarón capturado; 60 a 120 especies/l). No se utilizó oxígeno artificial.

\section{- Controles de Talla - Mortalidad}

En el área de siembra se procedió a abrir los embalajes, anotándose la mortalidad presentada en cada caja. Luego se extrae una muestra aleatoria de la semilla $(5 \%)$, registrándose solo la talla total del especímen (medida del rostrum al telson).

\section{- Aclimatación - Siembra - Densidad}

Las post larvas sembradas son aclimatadas con aguas del nuevo hábitat, agregándose lentamente agua de río en los embalajes que contienen las post larvas; este recambio dura entre 8 a 12 minutos. La etapa de siembra concluye cuando los especímenes inician su desplazamiento hacia las orillas del cauce y se distribuyen en el nuevo hábitat hídrico.

\section{CONTROLES ABIÓTICOS}

\section{a. Temperatura}

Se utilizó un termómetro de mercurio calibrado entre 0 a $50^{\circ} \mathrm{C}$ con una aproximación de $1^{\circ} \mathrm{C}$.

\section{b. Oxígeno Disuelto}

Mediante análisis de título volumétrico (método de Winkler Modificado), sugerida por NAVA (1974).

\section{c. Anhidrido Carbónico $\left(\mathrm{CO}_{2}\right)$}

Basado en el método citado por APHA, NAVA y WPCF (1976) y LAEVASTU (1971), empleando el indicador de fenolftaleina $0,5 \%$ y como titulador el $\mathrm{NaOH}$ N44.

\section{d. Potencial de Hidrógeno $(\mathrm{pH})$}

Se utilizó un potenciómetro portátil marca "Hach", de 0,5 unidades de precisión.

\section{RESULTADOS Y DISCUSIÓN}

\section{ÁREA DE UBICACIÓN}

El río Locumba y el río Sama son cuencas de la región tacneña, pertenecen a la vertiente occidental de los andes peruanos; ambos recursos hídricos tienen régimen semi constante, no llegando normalmente a desembocar sus aguas en el mar. Los 2 ríos poseen caudal casi constante solamentente hasta su zona media (puente Camiara - Locumba y represa Yarascay - Sama).

\section{ZONAS DE EXTRACIÓN}

Las semillas de camarón (Cryphiops caementarius) fueron extraídas en 02 zonas, con hábitat totalmente distintas :

\section{- Estuario de Ite}

Pequeña laguna formada en una explanada de la zona norte de la desembocadura del río Locumba, es un área que abarca una extensión entre 15 a 20 ha, paralelo a la línea costera que está delimitado por un acantilado de arenisca y grava, lo cual le proporciona una micro ecología especial. Se encuentra afecto a la contaminación directa de los relaves mineros y es depositario de los residuos sólidos vertidos en el río Locumba.

El estuario de Ite, es una laguna remanente formada por las filtraciones de agua de la Irrigacion de Ite, ubicada en la ladera cercana y superior de esta zona. El ambiente hídrico contiene una excesiva cantidad de post larva de camarón de río (Cryphiops caementarius), cuyo stock es probable que presente ciertas limitaciones genéticas respecto a condicionantes de crecimiento, movilidad y de talla, adquirida por la especíe en una zona ecológica desfavorable y que frecuenta normalmente. 


\section{- Cerro Colorado (río Sama)}

Es una zona ubicada en el tramo final del río Sama, con terreno accidentado, ondulante y de difícil acceso para la extracción de la semilla. En temporada de verano es notorio la abundancia de semillas de camarón. Presenta mayor uniformidad poblacional de semillas que el estuario de Ite pero alberga menor densidad de carga.

\section{ZONAS DE SIEMBRA}

Las zonas de siembra son 04 áreas convenientemente adecuadas para este fin; tres están ubicadas en el río Sama (zona Intermedia, Yarascay y Coruca) y uno en el río Locumba (Chipe), (Cuadro 1).

\section{DENSIDADES DE SIEMBRA}

Según los cálculos del presente proyecto, las densidades mínimas de siembra deben alcanzar cantidades entre 60000 camarones en el río Locumba y 40000 camarones en el río Sama; además la densidad poblacional promedio a mantenerse es de 0,5 camarones $/ \mathrm{m}^{2}$ de río.

Estudios poblacionales realizados en el río Locumba por CHIPOCO (1990), manifiesta que se requiere aproximadamente 66000 semillas de camarón para repoblar un tramo de $28,5 \mathrm{~km}$ de rio (densidad 0,5 camarones $/ \mathrm{m}^{2}$ ) y ORDETAM (1980) refiere la necesidad de utilizar 47145 semillas.

En el río Sama (1995), la Dirección Regional de Pesquería de Tacna introdujo 75000 camarones (talla $2,2 \mathrm{~cm}$ ).

\section{CANTIDADES RECOLECTADAS - INTRODUCIDAS}

En el Cuadro 1 se aprecia las cantidades de semillas que fueron recolectadas e introducidas. De la zona de Cerro Colorado (río Sama) fue posible recolectar 10800 post larvas con una talla que varió entre 2,4 a $3,2 \mathrm{~cm}$ y una talla promedio de $2,87 \mathrm{~cm}$. Del estuario de Ite se logró extraer y transportar 6500 semillas con talla promedio entre 3,0 a $3,5 \mathrm{~cm}$, especímenes relativamente más grandes que los recolectados en Cerro Colorado.

En el río Sama logró introducirse una cantidad total de 14000 semillas ( 9600 procedente de Cerro Colorado y 4400 del estuario de lte); en el río Locumba la cantidad sembrada fue de 3300 camarones $(1200$ de Cerro Colorado y 2100 de Ite).

En el presente proyecto logró efectivizarse una siembra total real de 17300 semillas de camarón (Cryphiops caementarius) para ambos ríos, con talla promedio que variaban entre 2,87 y $3,22 \mathrm{~cm}$.

\section{VARIACIÓN DE LA SEMILLA DURANTE EL TRANSPORTE}

Teniendo en consideración una muestra poblacional aleatoria $(n=50)$ de solamente 02 viajes de transporte, se puede apreciar la variación existente con respecto a la talla de la semilla transportada (Cuadro 2).

Las larvas procedentes del estuario de Ite, mantienen tallas entre 2,4 a $4,6 \mathrm{~cm}$, con promedios de $3,42 \mathrm{~cm}$, con un rango de mayor variación entre los especímenes.

Las semillas transportadas de la zona de Cerro Colorado (río Sama) tienen variaciones máximas de $0,6 \mathrm{~cm}$, con tallas promedios de $2,73 \mathrm{~cm}$, lo cual indica una mejor uniformidad poblacional de las post larvas extraídas.

\section{ANÁLISIS FÍSICO QUÍMICOS}

\section{a. En la Zona de Recolección (Cuadro 3)}

\section{- Estuario de Ite}

Los análisis de agua realizados en la zona de Ite muestran ligeras variaciones que existen entre la temperatura del agua y la del medio ambiente (rangos entre 22,7 a $24,1^{\circ} \mathrm{C}$ ). Los valores de oxígeno disuelto son bajos, oscilan entre 5,0 a 5,8 ppm; el anhídrido cárbonico varía con niveles medianamente altos de 9,6 a $11,0 \mathrm{ppm}$. Los rangos de $\mathrm{pH}$ indican que son aguas de procedencia semi ácida y con salinidades entre 5,4 a 6,8 o/oo (aguas salobres), dada por la existencia de abundante materia orgánica y la cercania al mar.

\section{- Cerro Colorado (Sama)}

Los análisis de agua coinciden solamente en las temperaturas delagua y del medio ambiente (variación entre ellos es de 22,8 a $24,7^{\circ} \mathrm{C}$ ). El oxígeno alcanza un valor máximo de $8,0 \mathrm{ppm}$ y 7,6 ppm de índice 
máximo de bióxido de carbono. Estas aguas mantienen valores casi neutros de $\mathrm{pH}$, con rango mínimo de variación $(0,2)$ y contenidos bajos de salinidad, lo cual refleja que son aguas de río costeño y con buen grado de movimiento.

Las zonas de extracción de post larvas muestran ligeras variaciones químicas de sus aguas, presentando mejores condiciones abióticas la zona de Cerro Colorado. Aún en las condiciones extremas del estuario de Ite, el camarón se adapta y sobrevive a las inclemencias del medio.

\section{b. En la Zona de Siembra (Cuadro 4)}

\section{- Río Locumba}

Las aguas del río Locumba mantienen variaciones mínimas de temperatura a través de la mayor parte del cauce medio (desde el puente de Locumba hasta Chipe), el rango es de 24,2 a $25,2^{\circ} \mathrm{C}$. Los niveles de oxígeno disuelto se mantienen estables (promedio 7,5 ppm) y el de bióxido de Carbono fluctua entre 8,4 a 11,6 ppm; con un pH promedio de 7,5 y una salinidad de 2,20/00. Los índices de bicarbonato están alrededor de 4,0 meq/l, no presentando carbonato.

Este río fue el medio ecológico habitual de grandes poblaciones de camarones; pero los factores contaminantes hídricos no controlados, procedentes de los relaves mineros arrojados en la zona cercana a la desembocadura, exterminaron la especie.

\section{- Río Sama}

Durante el proceso de siembra la temperatura ambiental y la temperatura del agua del río Sama mantienen idénticas condiciones (variación extrema $=1,7^{\circ} \mathrm{C}$ ), con una transparencia buena. Presenta mayor índice de oxígeno que el río Locumba $(8,6$ a $9,0 \mathrm{ppm}$ ) y menor nivel de bióxido de carbono (promedio 8,6 ppm). Existe similitud entre los valores alcalinos de $\mathrm{pH}$, los rangos de salinidad (máximo 2,4 o/oo) y los controles de bicarbonato y carbonato.

\section{TIEMPO DE TRANSPORTE Y TASA DE MORTALIDAD}

El tiempo de transporte y las mortalidades ocurridas en cada viaje de transporte están expresadas en el Cuadro 5. Porcentualmente los valores de la mortalidad no son significativas, encontrándose dentro de un rango que oscila entre 0,1 a $0,5 \%$ de la población desplazada. Además la mortalidad de transporte de animales vivos está influenciada por el tipo de aparejo utilizado en la recolección del organismo, experiencia del operador, tamaño de la semilla, condiciones alimenticias, etc.

Trabajos similares fueron realizados por diferentes instituciones en la zona del proyecto, donde reportan mortalidades extremas en semillas de camarón hasta de $45 \%$ por viaje.

La distancia existente desde la zona de recolección hacia los lugares de siembra es relativamente corto (Cerro Colorado - Chipe), por ello el tiempo de transporte abarca períodos entre 1:20 horas y 3:00 horas. Otros movimientos masivos de post larvas de camarón (Cryphiops caementarius) fueron realizados por la Dirección Sub Regional de Pesquería de Tacna (1995), utilizando 14 horas continuas de transporte (río Camaná a río Sama) con mortalidades que no llegaron a 5,0\%.

\section{EVALUACIÓN BIOLÓGICA PRELIMINAR}

Viajes de inspección y evaluación biológica efectuadas en la zona de estudio, reportan que las semillas introducidas en el río Locumba alcanzaron las etapas juveniles y de adultos, con tallas mayores a $12 \mathrm{~cm}$; estos camarones fueron capturados ocasionalmente por pobladores del lugar o ubicados en los cauces secos de los canales de irrigación (Mirave, Chipe, Sagoyo, Locumba, etc).

Los monitorajes preliminares indican que se lograron respuestas biológicamente positivas en los cauces donde se desarrolló el proyecto de repoblación, estimándose que las cantidades poblacionales sembradas no llegan a cubrir las demandas naturales de reclutamiento poblacional.

\section{CONCLUSIONES}

- El Proyecto permitió la ubicación de zonas adecuadas para la extracción de semillas de Cryphiops caementarius y de zonas ecológicamente favorables donde pueden ejecutarse la siembra y repoblación del crustáceo.

- Durante la realización del proyecto se logró movilizar una cantidad total de 17300 semillas de camarón de río, de las que 14000 semillas fueron introducidas 
en el río Sama y 3300 especímenes en el río Locumba; las tallas promedio oscilaron entre 2,87 y $3,22 \mathrm{~cm}$ respectivamente.

- La densidad promedio de siembra para ambos ríos fue de 0,5 camarones $/ \mathrm{m}^{2}$ de río, reportándose mortalidades en el transporte durante el movimiento de semillas entre 0,1 a $0,5 \%$ de la población. El tiempo de transporte varió entre 1:20 horas a 3:00 horas, para distancias no mayores a $190 \mathrm{~km}$ entre la zona de recolección y de siembra.

- Los factores físico químicos de las cuencas del río Sama y Locumba, indican valores semejantes en las temperaturas ambiental y del agua, $\mathrm{pH}$ y la salinidad del agua; existen variaciones significativas respecto

\section{REFERENCIAS BIBLIOGRÁFICAS}

CORDETACNA - MIPE (1984). Sub Proyecto "Siembra y Resiembra de Camarón". Convenio Corporación Departamental de Desarrollo de Tacna - Ministerio de Pesquería, DIREPE XII, Tacna, Perú. pp. 60.

CHIPOCO S. (1990). Transporte y Repoblación del Camarón de Río (Cryphiops caementarius) en el río Locumba - Tacna. Trabajo Informe. U.N.J.B.G. - Tacna.

ELIAS, H.J. (1972). Nota Preliminar sobre la Crianza del Camarón de Río Cryphiops caementarius. Documenta, Organo Informativo Técnico Científico, Ministerio de Pesquería. Lima - Perú № 22, pp. 42 - 49. a las condiciones de oxígeno (7,4 a $9,0 \mathrm{ppm})$ y $\mathrm{CO}_{2}$ (8,2 a 11,6 ppm), carbonatos (3,6 a 4,0 meq/l). En ambos tipos de agua no fue detectada la presencia de carbonatos (ppm).

\section{AGRADECIMIENTO}

- AlaDirección SubRegional dePesquería de Tacna, por el apoyo técnico - logístico, participando en forma conjunta en varias campañas de resiembra de camarones (Cryphiops caementarius) y en programas de capacitación técnica sobre crianza de este decápodo en la zona de Coruca, Tacna.
MIPE (1982). I Panel sobre Crustáceo del Perú. Dirección General de Extracción, Publicación Especial № 03. Marzo, Lima - Perú. pp. 181.

NAVA, C.H. (1974). Alimentación Artificial bajo diferentes niveles de proteina en Crianza Intensiva de Tilapia rendalli B. (Melanopleura). Tesis U.N.A. "La Molina", Prog. Acad. de Pesquería. Lima - Perú. pp. 129.

Cuadro 1. Áreas de Recolección y lugar de Siembra de las Semillas de Camarón de Tacna.

\begin{tabular}{|c|c|c|c|c|c|}
\hline \multirow{2}{*}{$\begin{array}{l}\quad N^{\circ} \\
\text { DE VIAJE }\end{array}$} & \multirow{2}{*}{$\begin{array}{l}\text { LUGAR DE } \\
\text { SIEMBRA }\end{array}$} & \multicolumn{4}{|c|}{ LUGAR DE RECOLECCIÓN } \\
\hline & & $\begin{array}{l}\text { CERRO } \\
\text { COLORADO }\end{array}$ & $\begin{array}{l}\text { TALLA } \\
\text { (cm) }\end{array}$ & ITE & $\begin{array}{c}\text { TALLA } \\
\text { (cm) }\end{array}$ \\
\hline 1 & $\begin{array}{l}\text { Río Sama } \\
\text { parte media }\end{array}$ & 3200 & 3.1 & 1200 & 3.5 \\
\hline 2 & $\begin{array}{l}\text { Río Sama } \\
\text { Coruca }\end{array}$ & 2600 & 2.4 & 1800 & 3.2 \\
\hline 3 & $\begin{array}{l}\text { Rio Sama } \\
\text { Represa Yarascay }\end{array}$ & 3800 & 2.8 & 1400 & 3.2 \\
\hline 4 & $\begin{array}{l}\text { Rio Locumba } \\
\text { Chipe }\end{array}$ & 1200 & 3.2 & 2100 & 3.0 \\
\hline \multicolumn{2}{|c|}{$\begin{array}{l}\text { TOTAL DE SEMILLAS } \\
\text { PROMEDIO TALLA }(\mathrm{cm})\end{array}$} & 10800 & 2.87 & 6500 & 3.22 \\
\hline
\end{tabular}

Fuente: Elaboración propia. 
Cuadro 2. Variación de las tallas en las Semillas de Camarón de río muestreadas en la zona de recolección $(n=50)$.

\begin{tabular}{|c|c|c|c|c|c|}
\hline No & ITE & CERRO COLORADO & No & ITE & CERRO COLORADO \\
\hline 1 & 3,7 & 2,9 & 14 & 2,9 & 2,5 \\
\hline 2 & 3,2 & 2,8 & 15 & 4,2 & 2,8 \\
\hline 3 & 2,8 & 2,4 & 16 & 4,6 & 2,4 \\
\hline 4 & 2,4 & 2,4 & 17 & 3,8 & 3,0 \\
\hline 5 & 3,8 & 3,0 & 18 & 3,6 & 3,0 \\
\hline 6 & 2,6 & 2,4 & 19 & 4,6 & 3,0 \\
\hline 7 & 2,4 & 2,8 & 20 & 3,7 & 2,8 \\
\hline 8 & 4,3 & 2,8 & 21 & 3,2 & 2,9 \\
\hline 9 & 3,8 & 3,0 & 22 & 3,1 & 3,0 \\
\hline 10 & 4,1 & 2,4 & 23 & 3,0 & 2,8 \\
\hline 11 & 3,6 & 2,5 & 24 & 2,8 & 3,0 \\
\hline 12 & 3,8 & 2,6 & 2,5 & 2,9 & 2,7 \\
\hline 13 & 2,6 & 2,9 & & \\
\hline
\end{tabular}

\begin{tabular}{|l|c|c|}
\hline \multicolumn{1}{|c|}{ DATOS ESTADÍSTICOS } & ITE & CERRO COLORADO \\
\hline Promedio (cm) & 3,42 & 2,73 \\
Desv. Stándar (S) & 0,662696511 & 0,235442845 \\
Varianza (s) & 0,649307323 & 0,230685933 \\
Sx & 85,50 & 68,20 \\
Sx & 302,95 & 187,38 \\
\hline
\end{tabular}

Fuente: Elaboración propia.

Cuadro 3. Análisis Físico Químicos del área de recolección de Semilla de Tacna.

\begin{tabular}{|c|c|c|c|c|c|c|c|c|}
\hline \multirow{2}{*}{$\begin{array}{l}\text { ANALISIS } \\
\text { CONTROL }\end{array}$} & \multicolumn{8}{|c|}{ NUMERO DE VIAJE } \\
\hline & ITE & $\begin{array}{c}1^{9} \\
\text { C.C. }\end{array}$ & ITE & $\begin{array}{c}2^{\circ} \\
\text { C.C. }\end{array}$ & ITE & $\begin{array}{c}3 \circ \\
\text { c.c. }\end{array}$ & ITE & $\begin{array}{r}4^{\circ} \\
\text { C.C. }\end{array}$ \\
\hline Tem Amb. & 22,7 & 23,2 & 24,3 & 23,6 & 24,2 & 23,3 & 24,1 & 23,2 \\
\hline Tem Agua & 24,3 & 24,7 & 23,5 & 24,4 & 23,8 & 23,5 & 24,0 & 22,8 \\
\hline Transp. & * & $\star \star$ & * & $*$ & * & $* * *$ & * & $* *$ \\
\hline Oxígeno & 5,0 & 7,8 & 5,2 & 8,0 & 5,4 & 7,6 & 5,8 & 8,1 \\
\hline $\mathrm{CO} 2$ & 10,6 & 6,4 & 9,6 & 7,4 & 11,0 & 7,6 & 10,2 & 6,8 \\
\hline $\mathrm{pH}$ & 6,6 & 7,8 & 6,4 & 7,6 & 6,2 & 7,8 & 6,0 & 7,8 \\
\hline Salinidad & 5,4 & 2,0 & 6,8 & 2,2 & 6,2 & 2,2 & 6,4 & 2,4 \\
\hline C.E. & 8,3 & 3,1 & 10,5 & 3,4 & 9,5 & 3,4 & 9,8 & 3,7 \\
\hline
\end{tabular}

Fuente: Elaboración propia.

C.C. $=$ Cerro Colorado
(*) Regular Tem Amb $\left({ }^{\circ} \mathrm{C}\right)$ Salinidad $(0 / 00)$
(*) Bueno Oxigeno (ppm) Cond Eléc $(\mathrm{ms} / \mathrm{cm})$
(..*) Muy Bueno $\mathrm{CO} 2(\mathrm{ppm})$ 
Cuadro 4. Análisis Físico Químicos controlados en aguas de los ríos Locumba y Sama de Tacna.

\begin{tabular}{|c|c|c|c|c|c|c|c|c|}
\hline \multirow{2}{*}{$\begin{array}{c}\text { ANALISIS } \\
\text { EFECTUADO }\end{array}$} & \multicolumn{4}{|c|}{ RIO LOCUMBA } & \multicolumn{4}{|c|}{ RIO SAMA } \\
\hline & $1^{\circ}$ & $2^{\circ}$ & $3^{\circ}$ & $4^{\circ}$ & $1^{\circ}$ & $2^{\circ}$ & 30 & $4^{\circ}$ \\
\hline Tem Amb. & 24,6 & 24,2 & 24,8 & 23,5 & 24,8 & 24,2 & 24,9 & 23,8 \\
\hline Tem Agua & 24,2 & 24,8 & 25,5 & 24,2 & 23,8 & 23,2 & 24,4 & 24,2 \\
\hline Transp. & $*$ & $* *$ & $\star *$ & $\star *$ & ** & * & $*$ & $*$ \\
\hline Oxígeno & 7,4 & 7,8 & 7,6 & 7,5 & 8,8 & 8,6 & 9,0 & 8,8 \\
\hline $\mathrm{CO} 2$ & 11,6 & 8,4 & 9,6 & 8,4 & 8,2 & 8,6 & 8,8 & 8,4 \\
\hline $\mathrm{pH}$ & 7,5 & 7,6 & 7,4 & 7,6 & 7,4 & 7,6 & 7,8 & 7,2 \\
\hline Salinidad & 1,8 & 2,2 & 2,2 & 2,2 & 2,4 & 2,2 & 2,4 & 2,0 \\
\hline C.E. & 2,8 & 3,4 & 3,4 & 3,4 & 3,7 & 3,4 & 3,7 & 3,1 \\
\hline Carbonato & 0,0 & 0,0 & 0,0 & 0,0 & 0,0 & 0,0 & 0,0 & 0,0 \\
\hline Bicarbonato & 4,0 & 4,1 & 3,8 & 4,0 & 3,6 & 3,8 & 4,0 & 3,8 \\
\hline
\end{tabular}

Fuente: Elaboración propia

$\begin{array}{lll}(*) \text { Regular } & (*) \text { Bueno } & \\ \text { Tem Amb }\left({ }^{\circ} \mathrm{C}\right) & \text { Oxigeno }(\mathrm{ppm}) & \mathrm{CO} 2(\mathrm{ppm}) \\ \text { Salinidad }(\mathrm{o} / \mathrm{oo}) & \text { Cond Eléc }(\mathrm{ms} / \mathrm{cm}) & \text { Carbonatos (ppm) } \\ \text { Bicarbonatos }(\mathrm{meq} / \mathrm{l}) . & & \end{array}$

Bicarbonatos (meq/l).

Cuadro 5. Tiempo de transporte y porcentaje de mortalidad según zona de recolección y siembra Semillas de Camarón de Tacna.

\begin{tabular}{|l|c|c|c|c|}
\hline \multirow{2}{*}{ LUGAR DE SIEMBRA } & \multicolumn{3}{|c|}{ LUGAR DE RECOLECCIÓN } \\
\cline { 2 - 5 } & \multicolumn{2}{|c|}{ CERRO COLORADO } & \multicolumn{2}{c|}{ ESTUARIO DE ITE } \\
\cline { 2 - 5 } & $\begin{array}{c}\% \\
\left.\mathbf{(}^{*}\right)\end{array}$ & $\begin{array}{c}\text { TIEMPO } \\
\text { (Horas) }\end{array}$ & $\begin{array}{c}\text { \% } \\
\left(^{*}\right)\end{array}$ & $\begin{array}{c}\text { TIEMPO } \\
\text { (Horas) }\end{array}$ \\
\hline $\begin{array}{l}\text { Río Sama } \\
\text { Parte Media }\end{array}$ & 0,2 & 1,20 & 0,5 & 2,00 \\
\hline $\begin{array}{c}\text { Rio Sama } \\
\text { Coruca }\end{array}$ & 0,1 & 2,20 & 0,3 & 3,00 \\
\hline $\begin{array}{c}\text { Río Sama } \\
\text { Represa Yarascay }\end{array}$ & 0,3 & 1,40 & 0,1 & 2,20 \\
\hline $\begin{array}{l}\text { Río Locumba } \\
\text { Chipe }\end{array}$ & 0,4 & 2,05 & 0,3 & 1,50 \\
\hline
\end{tabular}

Fuente: Elaboración propia.

(*) Porcentaje de Mortalidad. 